\title{
Reliability of the Pneumonia Severity Score (PSI) Index in Patients Diagnosed with COVID-19 Pneumonia to Determine Outpatient Discharge
}

\author{
Ahmet Burak Erdem ${ }^{1}$, Hakan Oğuztürk ${ }^{1, \star}$, Miray Tümer ${ }^{1}$, Bahattin Işık², \\ Afsin Emre Kayipmaz ${ }^{1}$, Semih Korkut ${ }^{3}$
}

\author{
${ }^{1}$ Ministry of Health, Ankara City Hospital \\ Emergency Medicine Clinic, Ankara, \\ Turkey \\ ${ }^{2}$ Dışkapı Yıldırım Beyazıt Education and \\ Research Hospital, Emergency Medicine \\ Clinic, Ankara, Turkey \\ ${ }^{3}$ Ministry of Health, Ankara, Turkey \\ *Correspondence \\ oguzturk@hotmail.com \\ (Hakan Oğuztürk)
}

\begin{abstract}
Objectives: Covid-19 infection, which has plagued the whole world, leads to deaths with a heavy pneumonia manifestation. Smoking, male gender and age stand out among the poor prognostic criteria of the disease. However, we still do not have a reliable scoring system for this disease. In this study, we aimed to find a reliable score at home discharge by using the Pneumonia severity index (PSI). Methods: Patients who came with suspected covid-19 in May were prospectively examined. The PSI scores of the patients at the time of admission to the emergency department were calculated. The PSI score of 161 patients included in our study was divided into 2 groups as $18-49$ years of age (group 1) and 50 and above (group 2). The clinical course of these patients was followed for 30 days. Data were associated with oxygen supplementation, need for intensive care, and mortality. Results: PSI score was significantly higher in group 1 males than females $(\mathrm{p}<0.001)$. There was no difference over 50 years old $(\mathrm{p}=0.571)$. The length of stay of group 2 patients was found to be significantly higher $(p=0.041)$. This may make us think that the treatment process becomes more difficult with age. The PSI scores of the 53 male patients were in Class 1 , and those of the 42 female patients were Class 1 . Among the patients who had the risk of 2 or more in Class 2 who were over the age of 50, 10 needed nasal oxygen, and 3 of these were transferred to Intensive Care Unit. Only one patient died. Conclusions: Patients over the age of 50 can be hospitalized if their PSI score is above 70, while patients under 50 can be followed up with home treatment. Group 2 patients without any additional disease can be followed up by telemedicine method.
\end{abstract}

\section{Keywords}

Covid-19, Pneumonia, PSI

\section{Introduction}

The novel type of Coronavirus (Covid-19), can result in severe respiratory failure and death. SARS-CoV and MERS$\mathrm{CoV}$, which are from the Coronavirus family, have previously caused outbreaks of severe respiratory failure and death [1]. The disease is more lethal in the elderly who have comorbid diseases (i.e. hypertension, diabetes mellitus, cardiovascular and cerebrovascular diseases) compared to younger patients and is more frequent in males than in females [2].

The Pneumonia Severity Index (PSI), has been used to determine the mortality in community-based pneumonia. Studies have been conducted to determine the relationship between PSI score and viral infections [3].

In order to better manage the Covid-19 pandemic, reliable scoring systems are needed to distinguish those patients who can be monitored at home as an outpatient vs those who require treatment in hospitals. The PSI score is a reliable way to determine the need for hospitalization for Community Based Pneumonia (CBP) [4]. In this study, we examined the reliability of the PSI scoring system for more efficient utilization of hospital beds during the Covid-19 pandemic.

\section{Material-Method}

\subsection{Study design and patient population}

This study was conducted prospectively with 294 patients admitted to the Emergency Department of Ankara City Hospital (Ankara/Turkey) in May 2020 with the suspicion of possible Covid-19 infection age 18 or above (Fig. 1). A total of 133 patients who had missing data, bacterial and non-Covid19 viral pneumonia, pregnant women, those without thoracic Computed Tomography (CT), without real-time polymerase chain reaction (RT-PCR) test, those under the age of 18 , and patients in whom Covid-19 infection were ruled out were excluded from the study. Patients whose tomography and RT- 


\section{A total of 294 patients}

\section{hospitalized to infection wards}

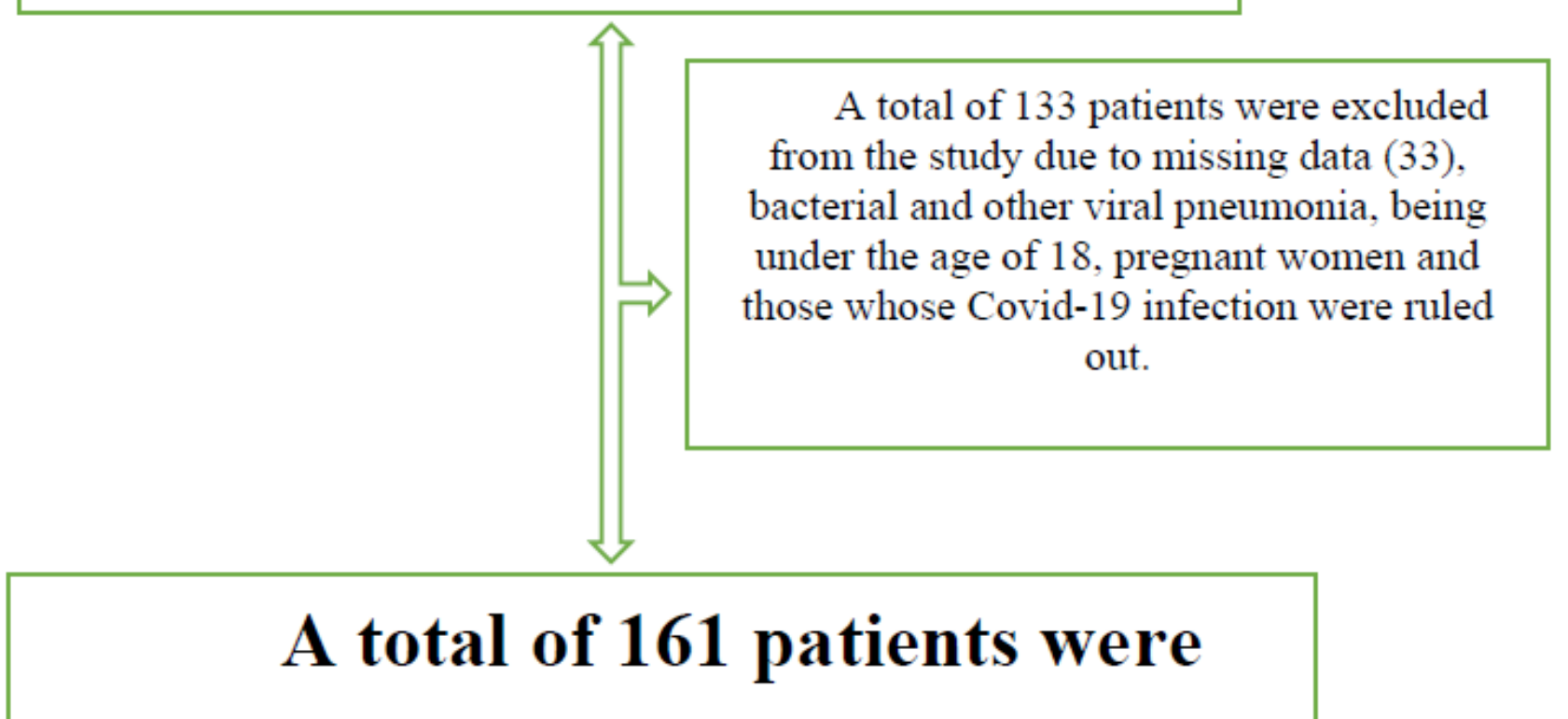

F I G U R E 1. Covid-19 infection flow chart.

PCR test were negative simultaneously were not considered as having a Covid-19 infection. We excluded patients who did not undergo lung tomography because it is more sensitive in diagnosing infiltrates than a routine plain chest X-ray. The 161 patients included in the study were followed for 30 days. The patients were divided into 2 groups according to their ages; 18 to 49: Group 1 and 50 or older; Group 2. Patients who required oxygen and respiratory support when they arrived in the emergency department and then were admitted to intensive care were not included in the study. These patients were hospitalized and treated irregardless of the PSI score. Our aim was to determine which patients with covid-19 infection would need oxygen support or intensive care using PSI scores.

The study was conducted with the approval of the Ethics Board of the Ministry of Health, Ankara City Hospital (E1-20582). The study was conducted in accordance with the Helsinki Declaration.

\subsection{Definitions}

The patients suspected of having Covid-19 infection who presented with fever, cough, shortness of breath, sore throat and who underwent thoracic CT formed the Study Group. The patients who had Thorax CT reports in the emergency department consistent with a Covid-19 viral infection (groundglass appearance, consolidation, paving stone appearance, air bronchogram, vascular expansion and bronchial changes in the medial and lower segments, in the form of a peribronchovascular distribution) were admitted to the infection diseases ward from the emergency department. The PSI scores of the patients in the emergency department were calculated using the following scoring system: age (the same in males, 10 points lower in females), 10 points for nursing home, 30 points for the presence of malignancy , 20 points for liver disease, heart failure, cerebrovascular disease and kidney disease 10 points each, BUN $\geq 30 \mathrm{mg} / \mathrm{dl}$ and $\mathrm{Na}<130 \mathrm{mg} / \mathrm{dl} 20$ points, 10 points for Glucose $\geq 250 \mathrm{mg} / \mathrm{dl}$ and $\mathrm{Htc}<30 \%$ each, change in consciousness, respiratory count $\geq 30 / \mathrm{min}$ and systolic blood pressure $<90 \mathrm{mmhg} 20$ points each, fever $<35^{\circ} \mathrm{C}$ or $\geq$ $40^{\circ} \mathrm{C} 15$ points, heart rate $\geq 125 / \mathrm{min}$ and pleural effusion 10 points each, arterial ph $<7.3530$ points, 10 points for $\mathrm{Pa} 02$ $<60 \mathrm{mmhg}$ and/or S02 $<90 \%$. The PSI scores were defined as Class $1(<50), 2(\leq 70), 3(71-90), 4(91-130)$ and $5(>$ $130)$; the higher scores were associated with increased clinical symptoms.

\subsection{Data collection}

The age, gender, radiological findings, PSI scores, RT-PCR results, length of hospital stay and 30-day mortality were recorded for each patient. The PSI scores at the time of the arrival at the emergency department were calculated according to age, comorbid diseases, clinical findings and laboratory data. The data were obtained from the hospital's data processing system. Those who needed oxygen support or those who were admitted to intensive care, were also recorded. The 30-day mortality and recurrent hospital readmissions of the patients were identified through the national patient follow-up system 
TA B L E 1. The demographic, PSI scores, CT, PCR and Comorbidity status of the patients.

\begin{tabular}{|c|c|c|c|c|c|}
\hline & & Total (n:161) & $\begin{array}{c}\text { Group } 1 \\
(n: \text { 94) }(18-49)\end{array}$ & Group 2 (n:67) (50+) & $\mathbf{p}$ \\
\hline Age, Median (IQR) & & $45(21)$ & $38(12)$ & $61(15)$ & $<0.001$ \\
\hline \multirow{2}{*}{ Gender, n(\%) } & Male & $92(57.1)$ & $53(56.4)$ & $39(58.2)$ & 0.817 \\
\hline & Female & $69(42.9)$ & $41(43.6)$ & $28(41.8)$ & \\
\hline PSI, Median (IQR) & & $44(27.5)$ & $34.5(15.3)$ & $64(22)$ & $<0.001$ \\
\hline $\mathrm{CT}, \mathrm{n}(\%)$ & Positive & $157(97.5)$ & $90(95.7)$ & $67(100)$ & 0.142 \\
\hline PCR, n $(\%)$ & Positive & $62(38.5)$ & $38(40.4)$ & $24(35.8)$ & 0.623 \\
\hline Time, Median (IQR) & & $7(5)$ & $6.5(4)$ & $7(5)$ & 0.041 \\
\hline Pleural effusion, n (\%) & Yes & $4(2.5)$ & $0(0)$ & $4(6)$ & 0.028 \\
\hline Nasal O2, n (\%) & Yes & $9(5.6)$ & $1(1.1)$ & $8(11.9)$ & 0.003 \\
\hline Hypergylcemia, n (\%) & Yes & $3(1.9)$ & $0(0)$ & $3(4.5)$ & 0.07 \\
\hline Low Htc, n (\%) & Yes & $3(1.9)$ & $1(1.1)$ & $2(3)$ & 0.571 \\
\hline HT, n (\%) & Yes & $37(23)$ & $6(6.4)$ & $31(46.3)$ & $<0.00$ \\
\hline DM, n (\%) & Yes & $29(18)$ & $7(7.4)$ & $22(32.8)$ & $<0.001$ \\
\hline $\mathrm{HF}, \mathrm{n}(\%)$ & Yes & $7(4.3)$ & $1(1.1)$ & $6(9)$ & 0.021 \\
\hline Malignancy, n (\%) & Yes & $6(3.7)$ & $1(1.1)$ & $5(7.5)$ & 0.083 \\
\hline CRF, n (\%) & Yes & $5(3.1)$ & $1(1.1)$ & $4(6)$ & 0.161 \\
\hline COPD, n (\%) & Yes & $4(2.5)$ & $0(0)$ & $4(6)$ & 0.028 \\
\hline Other, n (\%) & & $2(1.2)$ & $0(0)$ & $2(3)$ & 0.172 \\
\hline
\end{tabular}

CT: Computed tomography, HT: hypertension, DM: Diabetes mellitus, HF: Hearth failure, CRF: Chronic renal failure, COPD: Chronic obstructive pulmonary disease; Htc:hematocrit, PSI:Pneumonia Severity Index.

e-nablz). The patients were again screened within 30 days after discharge. The data were recorded by 2 specialist doctors (emergency medicine), and a third specialist doctor checked these data.

\subsection{Outcomes}

Patients who had CT findings consistent with Covid-19, even if the RT-PCR was negative who exhibited clinical findings, and patients with RT-PCR positive results were evaluated and treated as Covid-19 pneumonia.

\subsection{Statistical Analysis}

The data were analyzed in SPSS for Windows ${ }^{\circledR} 22.0$. The compliance of the data with normal distribution was determined with the Kolmogorov-Smirnov Test. Median and Interquartile Range (IQR) were used when the data failed to comply with a normal distribution. Mann Whitney-U test was used in the analysis of the nonparametric data. In comparison of the frequency data between two categorical variables, the Chi-Square and Fisher's Exact Test were used. Spearman Correlation Analysis was used to compare the quantitative data with each other. The value of $p<0.05$ was considered statistically significant in the analyses.

\section{Results}

The median age of the patients was 45 (IQR:21), and $57.1 \%$ of the patients were male. No gender differences were detected
TA B L E 2. Distribution of PSI scores by gender.

\begin{tabular}{lccc} 
PSI & $\begin{array}{c}\text { Male Median } \\
\text { (IQR) }\end{array}$ & $\begin{array}{c}\text { Female Median } \\
(\text { IQR) }\end{array}$ & p \\
Group 1 & $38(13.5)$ & $28(14)$ & $<0.001$ \\
Group 2 & $64(27)$ & $64.5(20.8)$ & 0.571 \\
\hline
\end{tabular}

between groups $(\mathrm{p}=0.817)$. The PSI score of the patients over the age of 50 (Group 2) was significantly higher $(\mathrm{p}<$ 0.001 ). A total of $97.5 \%$ of the cases had a CT, and $38.5 \%$ had a positive PCR. No differences were detected between the groups in terms of CT and PCR positivity $(\mathrm{p}=0.142, \mathrm{p}=$ 0.623). We performed imaging in patients with simple upper respiratory tract symptoms, a history of contact with someone with a positive RT-PCR test, but who were asymptomatic.

The hospitalization time of Group 2 patients was significantly longer $(p=0.041)$. The incidence of pleural effusions, need for nasal oxygen, Hypertension (HT), Diabetes Mellitus (DM), Heart Failure (HF), and Chronic Obstructive Pulmonary Disease (COPD) were significantly higher in Group 2 $(\mathrm{p}<0.05)$. No differences were detected between the groups in terms of hyperglycemia, decrease in hematocrits (Hct), frequency of malignancy, frequency of chronic renal failure $(\mathrm{CRF})$ and other diagnoses (Table 1) $(\mathrm{p}>0.05)$.

The PSI score of the male patients under the age of 50 (Table 2 ) was significantly higher $(\mathrm{p}<0.001)$. No statistically significant differences were detected between the PSI scores of the patients over the age of 50 ( $p>0.571)$.

A positive correlation was detected between age $(\mathrm{p}<0.001)$, 
TA B L E 3. PSI scores and hospitalization durations.

\begin{tabular}{lcccccccc} 
& \multicolumn{2}{c}{ Age } & \multicolumn{2}{c}{ PSI } & \multicolumn{3}{c}{ DURATION } \\
& $\mathrm{r}$ & & $\mathrm{p}$ & $\mathrm{r}$ & $\mathrm{p}$ & $\mathrm{r}$ & $\mathrm{p}$ \\
\hline Age & \multicolumn{2}{c}{1} & & $0.905^{* *}$ & $<0.001$ & $0.171^{*}$ & 0.03 \\
PSI & $0.905^{* *}$ & $<0.001$ & \multicolumn{2}{c}{1} & & 0.155 & 0.05 \\
\hline DURATION & $0.171^{*}$ & 0.03 & 0.155 & & 0.05 & \multicolumn{2}{c}{1} & \\
\hline
\end{tabular}

TA B L E 4. Distribution of PSI score classification by gender.

\begin{tabular}{|lccccc|} 
PSI & $\begin{array}{c}\text { CLASS 1 } \\
(<\mathbf{5 0}) \mathbf{n}\end{array}$ & $\begin{array}{c}\text { CLASS 2 } \\
(<\mathbf{7 0}) \mathbf{n}\end{array}$ & $\begin{array}{c}\text { CLASS 3 } \\
(\mathbf{7 1}-\mathbf{9 0}) \mathbf{n}\end{array}$ & $\begin{array}{c}\text { CLASS 4 } \\
(\mathbf{9 1}-\mathbf{1 3 0}) \mathbf{n}\end{array}$ & $\begin{array}{c}\text { CLASS 5 } \\
(>\mathbf{1 3 0}) \mathbf{n}\end{array}$ \\
\hline Male & 53 & 23 & 10 & 5 & 1 \\
\hline Female & 42 & 18 & 7 & 2 & 0 \\
\hline
\end{tabular}

PSI score and the length of hospitalization $(p=0.030)$ (Table $3)$.

The PSI scores of the 53 male patients were in Class 1, and those of the 42 female patients were Class 1 (Table 4). Among the patients who had the risk of 2 or more in Class 2 who were over the age of 50,10 needed nasal oxygen, and 3 of these were transferred to the Intensive Care Unit. Three of our patients transferred to intensive care were more than 50 years old (64, 79,91 ). One male patient who had a PSI score of 121 died after being admitted to the Intensive Care Unit. Nasal oxygen was needed in only one male patient who was at the age of 38 with a Class 1 risk.

In the chest tomography performed, 154 patients had ground glass density infiltration areas, mostly located in peripheral and/or sometimes central different foci. Of these patients, 18 were accompanied by consolidation, 5 by air bronchogram and consolidation, 3 by paving stone view, 2 by septal thickening, 2 by budding tree view, 1 by mosaic attenuation, 1 by halo sign, and 1 by reverse halo sign. 3 patients had atypical lesions. These were in the form of increased sentiacinar density, soft tissue density lesion without air bronchogram, thickening and secretion in the bronchial walls. There were no findings in 4 patients on tomography. 3 atypical and asymptomatic 4 patients were included in the study because their RT-PCR results were positive (Table 5).

TA B L E 5. Chest tomography findings of the patients.

\begin{tabular}{lcc} 
Findings & $\mathbf{n}$ & $\mathbf{\%}$ \\
\hline Areas with ground glass density & 154 & 95.65 \\
Atypical findings & 3 & 1.86 \\
Normal görünüm & 4 & 2.49 \\
\hline
\end{tabular}

The distribution of PSI values by age is summarized in Fig. 2.

\section{Discussion}

PSI consists of 20 variables. Class $1-3$ is considered low risk because of the low mortality rate, and Class $4-5$ is considered as high risk because of the high mortality rate. In a study conducted in 8 emergency departments in France, Class 1 and 2 cases were treated in $\mathrm{CBP}$ as outpatients, and found the results to be reliable [5]. Since the Covid-19 infection is a new Coronavirus infection, data on clinical course and treatment are limited. It can be asymptomatic in the form of a simple upper respiratory tract infection, or result in respiratory failure due to severe pneumonia and death [6]. For this reason, reliable patient risk classification systems are needed. In our study, we investigated the PSI scoring system to predict hospital discharge. In our patients, during the 30-day mortality followup, mortality was observed only in one patient. This was consistent with data from the literature [7]. We determined that these patients did not require readmission to the hospital. The median value of the PSI in Group 2 patients was 64 . In the clinical follow-up of these patients, the need for nasal oxygen $(\mathrm{p}<0.05)$ occurred a few days after hospitalization compared to Group 1 patients, and only 3 patients needed intensive care.

Shi et al. performed a host risk classification consisting of 3 factors, age, gender and hypertension in 487 patients, and found that the presence of male gender, being over the age of 50, and hypertension were risk factors [6]. Our study was consistent with Shi et al. results in that 2 of our patients who subsequently required intensive care were over 50 years of age and 2 of them were male. Kim et al. [3] and Zhaou et al. showed the importance of age in terms of prognosis in their study on PSI and viral pathogens [8]. In our study, the need for nasal oxygen support was greater in patients over 50 years of age. Liu et al. compared elderly and young and middleage patients, and found the PSI scores of older patients were significantly higher [2]. The clinical hospitalization decision of CBP patients with PSI scores differed amongst previous studies. Many patients in the low-risk class were treated with the suspicion of hypoxia [7]. We excluded the patients who needed oxygen support in the emergency department, and those admitted to the Intensive Care Unit. The need for hypoxia and intensive care that developed in Group 2 patients can be explained by the deterioration of ventilation as a result of the inflammatory response caused by inflammation which can result in cytokine storm [9].

Previous studies have shown that increased age, male gender, smoking, HT, DM, cardiovascular and lung diseases are risk factors for prognosis [9-11]. Group 2 patients had significant differences in terms of HT, DM, HF and COPD. In this study, a positive relationship was detected between PSI score and age and discharge times. As the score and age 


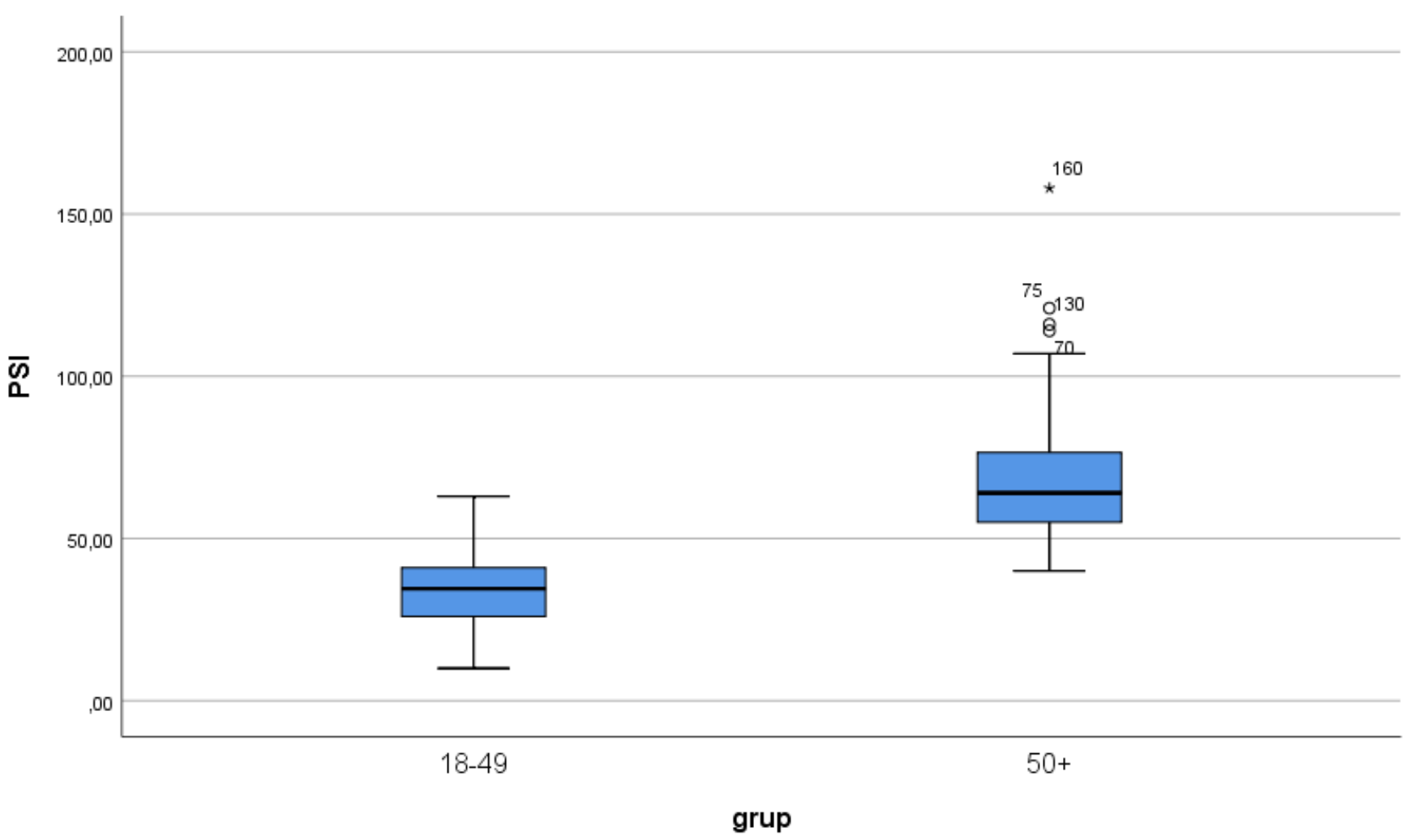

F I G U R E 2. Group 1 and 2's PSI score distribution.

increased, the length of hospitalization of our patients also increased. The decision to discharge these patients was made using clinical recovery and a negative RT-PCR result on at least 2 occasions. The increased duration of discharge times in Group 2 patients may be explained by the high-risk factors for Covid-19 pneumonia in these patients. The PSI score of male patients was largely compatible with Class 1 scores. The male patients in Class 2 and above needed more intensive care. One 91-year-old male patient who had Class 4 risk needed intensive care, and died in this unit. The other two patients were over 50 years of age, and had COPD. Most of our female patients had a Class 1 PSI score, and these patients did not require intensive care. Only one female patient with COPD who had a PSI score of 54 needed intensive care after 1 week. The PSI scores were significantly higher in our male patients who were under 50 years of age than in female patients. In the light of these data, our study shows that more attention should be paid to the prognosis of male patients.

PSI is a scoring system and is calculated according to age, gender, nursing home stay, comorbid disease, urea, glucose, sodium, hematocrits, consciousness status, vital signs, pleural effusions and oxygenation level [3, 4, 7]. It has been shown in laboratory studies conducted in Covid-19 patients that lymphopenia, increased Lactate Dehydrogenase (LDH) and C-Reactive Protein (CRP) values are associated with poor prognosis [12, 13]. Shi [6], Hu [14] and Ji [15] evaluated Covid-19 prognosis with a 3-Host risk scoring system, rapid score systems (REMS and MEWS) and CALL (Comorbidity, Age, Lymphocyte and LDH) scores, which correlated with the prognosis of Covid-19 patients. Although these scoring systems were easy to use, they lacked all of the Covid-19 risk factors. The PSI score can provide a better measurement of prognosis with simultaneous evaluation of more parameters. However, smoking, CRP, LDH and lymphopenia, which are among the risk factors in Covid-19, are missing. In subsequent studies using PSI, more accurate results can be achieved by replacing staying in a nursing home with smoking, replacing sodium with LDH-CRP, and the lymphocyte value instead of hematocrit.

\section{Limitations}

The fact that the whole study group did not consist of patients with simultaneous positive RT-PCR and tomography was a major limiting factor. Another factor was that a group of intensive care patients was not included in this study. Our intention was that this group already met requirements for hospitalization. We wanted to determine whether patients who are treated in wards but who are in good general clinical condition could benefit from PSI prognostic scoring to guide the triage, and especially, during the time when there are a shortage of beds in hospitals. We were able to follow and treat every possible patient. Among these patients, most of them did not need respiratory and oxygen support, such that they were eligible for outpatient monitoring.

\section{Conclusion}

The present study showed that for patients under 50 years with a Class 1 PSI score, such patients are suitable for home follow-up. If the PSI score of patients, who are over 50 years of age and who have comorbid diseases, is 70 or more, 
it is better to monitor them by hospitalization. Because of the uncertainty in treatment in Covid-19, cytokine storm, the prolongation of RT-PCR negative time as the age increases $(p=0.030)$, a PSI score above 70 points in patients in need of intensive care, and the inability to predict hypoxia that may develop; are important reasons for hospital follow-up in these patients. Class 2 patients, on the other hand, may be monitored at home with the Tele Medicine Method if they do not have comorbid diseases. Finally, if the PSI scores and the risk factors identified for Covid-19 are combined, this has the potential to be another useful predictor of clinical outcomes in these patients.

\section{ACKNOWLEDGEMENTS}

Thank numerous individuals participated in this study.

\section{CONFLICT OF INTEREST}

There is no conflict of interest on the part of any author.

\section{REFERENCES}

[1] Chen N, Zhou M, Dong X, et al. Epidemiological and clinical characteristics of 99 cases of 2019 novel coronavirus pneumonia in Wuhan, China: a descriptive study. Lancet. 2020;395:507-513.

[2] Liu K, Chen Y, Lin R, et al. Clinical features of COVID-19 in elderly patients: A comparison with and middle-aged patients. J Infect. 2020;80:e14-e18.

[3] Kim M-A, Park JS, Lee CW, et al. Pneumonia severity index in viral community acquired pneumonia in adults. PLoS One. 2019;14:e0210102.

[4] Marcos PJ, Restrepo MI, González-Barcala FJ, et al. Discordance of physician clinical judgment vs. pneumonia severity index (PSI) score to admit patients with low risk community acquired pneumonia: a prospective multicenter study. J Thorac Dis. 2017;9:1538-1546.

[5] Renaud B, Coma E, Labarere J, et al. Routine Use of the Pneumonia Severity Index for Guiding the Site-of-Treatment Decision of Patient with
Pneumonia in the Emergency Department: A Multicenter, Prospective, Observational, Controlled Cohort Study. Clin Infect Dis. 2007;44:41-49.

[6] Shi Y, Yu X, Zhao H, et al. Host susceptibility to severe COVID-19 and establishment of a host risk score: findings of 487 cases outside Wuhan. Crit Care. 2020;24:108.

[7] Seymann, G, Barger K, Choo S, et al. Clinical judgment versus the pneumonia severity index in making the admission decision. J Emerg Med. 2008;34:261-268.

[8] Zhou F, Yu T, Du R, et al. Clinical course and risk factors for mortality of adult inpatients with COVID-19 in Wuhan, China: a retrospective cohort study. Lancet. 2020;395:1054-1062.

[9] Zheng Z, Peng F, Xu B, et al. Risk factors of critical \& mortal COVID19 cases: A systematic literature review and meta-analysis. J Infec. 2020;81:e16-e25.

[10] Liu W, Tao ZW, Wang L, et al. Analysis of factors associated with disease outcomes in hospitalized patients with 2019 novel coronavirus disease. Chin Med J (Engl). 2020;133:1032-1038.

[11] Deng Y, Liu W, Liu K, et al. Clinical characteristics of fatal and recovered cases of coronavirus disease 2019 (COVID-19) in Wuhan, China: a retrospective study. Chin Med J (Engl). 2020;133:1261-1267.

[12] Mo P, Xing Y, Xiao Y, et al. Clinical characteristics of refractory COVID-19 pneumonia in Wuhan, China. Clin Infect Dis. 2020. doi: 10.1093/cid/ciaa270.

[13] Henry BM, Oliveira MHS, Benoit S, et al. Hematologic, biochemical and immune biomarker abnormalities associated with severe illness and mortality in coronavirus disease 2019 (COVID-19): a meta-analysis. Clin Chem Lab Med. 2020;58:1021-1028.

[14] $\mathrm{Hu} \mathrm{H}$, Yao N, Qiu Y. Comparing rapid scoring systems in mortality prediction of critical ill patients with novel coronavirus disease. Acad Emerg Med. 2020;27:461-468.

[15] Ji D, Zhang D, Xu J, et al. Prediction for Progression Risk in Patients with COVID-19 Pneumonia: the CALL Score. Clin Infect Dis. 2020;71:13931399.

How to cite this article: Ahmet Burak Erdem, Hakan Oğuztürk, Miray Tümer, Bahattin Işı, Afsin Emre Kayipmaz, Semih Korkut. Reliability of the Pneumonia Severity Score (PSI) Index in Patients Diagnosed with COVID-19 Pneumonia to Determine Outpatient Discharge. Signa Vitae. 2021;17(2):48-53. doi:10.22514/sv.2020.16.0074. 